INPLASY

PROTOCOL

To cite: Fan et al. Jingui Shenqi

Pill for Nocturia Due to Nocturnal Polyuria A protocol for systematic review and meta-analysis. Inplasy protocol 2020110048. doi:

10.37766/inplasy2020.11.0048

Received: 11 November 2020

Published: 12 November 2020

Corresponding author:

Yong Jiang

jiangyong@pyztcm.com

Author Affiliation:

Basic Medical College, Chengdu University of TCM

Support: NSFC.

Review Stage at time of this submission: Preliminary

searches.

Conflicts of interest:

The authors have no conflict of interest to disclose.

\section{Jingui Shenqi Pill for Nocturia Due to Nocturnal Polyuria A protocol for systematic review and meta-analysis}

Fan, ZP1; Wang, ZD²; Deng, JS3; Jiang, Y4.

Review question / Objective: The purpose of this systematic review is to evaluate the efficacy and safety of JSP in the treatment of NP.

Condition being studied: The incidence of nocturia is high and will seriously affect patients' physical and mental health. Nocturnal polyuria is the most critical cause of nocturia, There are few drugs currently used to treat nocturia due to Nocturnal Polyuria (NP). The guide highly recommends only Desmopressin. There is an urgent need to find new drugs. Jingui Shenqi pill (JSP) is a Chinese patent medicine, It is widely used in China to treat NP. However, there is no evidence-based medical evidence to prove its safety and effectiveness. The purpose of this systematic review is to evaluate the efficacy and safety of JSP in the treatment of NP.

INPLASY registration number: This protocol was registered with the International Platform of Registered Systematic Review and Meta-Analysis Protocols (INPLASY) on 12 November 2020 and was last updated on 12 November 2020 (registration number INPLASY2020110048).

\section{INTRODUCTION}

Review question / Objective: The purpose of this systematic review is to evaluate the efficacy and safety of JSP in the treatment of NP.

Condition being studied: The incidence of nocturia is high and will seriously affect patients' physical and mental health. Nocturnal polyuria is the most critical cause of nocturia, There are few drugs currently used to treat nocturia due to Nocturnal Polyuria (NP). The guide highly recommends only Desmopressin. There is an urgent need to find new drugs. Jingui Shenqi pill (JSP) is a Chinese patent 
medicine, It is widely used in China to treat NP. However, there is no evidencebased medical evidence to prove its safety and effectiveness. The purpose of this systematic review is to evaluate the efficacy and safety of JSP in the treatment of NP.

\section{METHODS}

Participant or population: Patients diagnosed with NP and aged $>18$ years old will be included, regardless of gender, economic status, or restrictions.

Intervention: The intervention group used only JSP or combined JSP with another active treatment, pharmacological or nonpharmacological.

Comparator: The control group used another active treatment or placebo or no treatment. The dosage and frequency will not be restricted.

Study designs to be included: All RCTs about JSP for NP will be included regardless of language. The following studies will be excluded: animal experiments, case series, quasi-RCTs, cell experiments, Case reports, non-RCTs.

Eligibility criteria: All RCTs about JSP for NP will be included regardless of language. The following studies will be excluded: animal experiments, case series, quasiRCTs, cell experiments, Case reports, nonRCTs. 2.3.2. Participants. Patients diagnosed with NP and aged $>18$ years old will be included, regardless of gender, economic status, or restrictions. 2.3.3. Types of interventions. The intervention group used only JSP or combined JSP with another active treatment, pharmacological or non-pharmacological. The control group used another active treatment or placebo or no treatment. The dosage and frequency will not be restricted.

Information sources: The following eight electronic databases, including PubMed, Cochrane Library, EMBASE, MEDLINE, the China National Knowledge Infrastructure, the Chinese Biomedical Literature Database, Cqvip Database, and Wanfang Data, will be comprehensively searched. To identify additional references, we will manually search the reference lists of primary studies and related reviews. We also will search the following resources to identify ongoing or completed clinical trials: Google scholar, Opengrey, Chinese Clinical Trial Registry, ClinicalTrials. gov, International Clinical Trials Registry Platform.

Main outcome(s): The primary outcomes include Nocturnal urine volume, the number of nocturnal voids, Nocturnal polyuria index (Nocturnal total urine volume /24h total urine volume).

Quality assessment / Risk of bias analysis: Two reviewers will use the Cochrane Collaboration's tool to assess methodological quality. We will evaluate the following seven aspects. Including random, blinding of participants and investigators, sequence generation, the blindness of outcome assessments, allocation concealment, selective outcome reporting, incomplete outcome data, and other biases. Each included study will be assessed as low, unclear, or high bias based on the results. Any discrepancies will be resolved by further discussing it with a third reviewer.

Strategy of data synthesis: For data analysis, we will use RevMan 5.3.0 provided by the Cochrane Collaboration. The chisquare test and 12 statistic will be used to assess the heterogeneity of similar studies. If $P \geq 0.05$ and $12 \leq 50 \%$, it is low heterogeneity. As a result, a fixed-effects model will be used. If $P<0.05$ and $I 2>50 \%$, there will be heterogeneity. a randomeffects model will be used. We will use an odds ratio(OR) with a $95 \%$ confidence interval $(\mathrm{Cl})$ to represent the enumeration data. We will express the measurement data by the mean difference(MD with 95\% Cl. A statistically significant difference is considered to be $P<0.05$. 
Subgroup analysis: To seek the potential source of heterogeneity, subgroup analysis, and sensitivity analysis will be performed based on various study characteristics such as study quality, location of study, publication date, trial publishing status, type of comparisons, history of pulmonary diseases, duration of treatment, frequency of delivery, age, gender.

Sensibility analysis: To seek the potential source of heterogeneity, subgroup analysis, and sensitivity analysis will be performed based on various study characteristics such as study quality, location of study, publication date, trial publishing status, type of comparisons, history of pulmonary diseases, duration of treatment, frequency of delivery, age, gender.

Country(ies) involved: China.

Keywords: Jingui Shenqi pill, Effectiveness, Safety, Nocturia, Nocturnal Polyuria, Systematic review.

Contributions of each author:

Author 1 - Zhipeng Fan Conceptualization; Methodology; Project administration; Writing - original draft; Writing - review \& editing.

Email: 156183951@qq.com

Author 2 - Zhaodi Wang Conceptualization; Investigation; Methodology; Validation; Writing - review \& editing.

Email: 13213968850@163.com

Author 3 - Jiashuai Deng Conceptualization; Project administration; Supervision.

Email: dy521561@outlook.com

Author 4 - Yong Jiang Data curation; Software.

Email: jiangyong@pyztcm.com 